\title{
Health promotion programme design and efficacy in relation to ageing persons with culturally and linguistically diverse backgrounds: a systematic literature review and meta-analysis
}

Qarin Lood ${ }^{1,2,3^{*}}$, Greta Häggblom-Kronlöf $f^{1,2,3+}$ and Synneve Dahlin-Ivanoff', $2,3+$

\begin{abstract}
Background: Health promotion has the potential to empower people to develop or maintain healthy lifestyles. However, previous research has visualised serious health and healthcare inequities associated with ageing, cultural affiliations and linguistic preferences. Therefore, this study was part of a larger health promotion project, set out to bridge barriers to health for ageing persons who have migrated to Sweden. More specifically, the present study aimed to elucidate the content and effects of multidimensional health promotion programmes in the context of ageing persons with culturally and linguistically diverse backgrounds.
\end{abstract}

Methods: Databases were systematically searched to identify relevant randomised controlled trial publications. All potentially relevant publications were assessed for relevance and design and after this screening, a final sample of eight publications could be included in the review. Those publications evaluated six different programmes and a mixed-methods approach to data analysis was applied, using a combination of narrative synthesis, meta-analyses and evidence grading.

Results: The findings suggest a multidimensional health promotion programme design for ageing persons with culturally and linguistically diverse backgrounds, involving culturally and linguistically modified activities and health information that should be provided by professionals with a person-centred approach. In addition, the meta-analyses revealed statistically significant effects in favour of health promotion on: general health, depression, mental health, physical health, and vitality. However, the evidence for the identified effects is low, and further research findings are likely to change the estimations.

Conclusions: The present study provides an aggregation of health promotion intervention research with older persons with culturally and linguistically diverse backgrounds; a group of people who are commonly excluded from research, and marginalised when it comes to health and healthcare. By visualising the core components of health promotion programmes with proven efficacy, the findings provide guidance for further explorations of how health promotion should be designed to minimise inequities in health.

Keywords: Aged, Emigrants and immigrants, Global health, Healthcare disparities, Minority health

\footnotetext{
*Correspondence: qarin.lood@neuro.gu.se

${ }^{\dagger}$ Equal contributors

1 Institute of Neuroscience and Physiology, Section for Health and

Rehabilitation, The Sahlgrenska Academy, University of Gothenburg, Box

455SE-405 30 Gothenburg, Sweden

${ }^{2}$ University of Gothenburg Centre for Person-Centred Care (GPCC),

Gothenburg, Sweden

Full list of author information is available at the end of the article
} 


\section{Background}

Health promotion has become a fundamental component of healthcare services aimed at older persons [1], and it has been well documented that multidimensional health promotion programmes commenced before the onset of poor health can have a large impact on their possibilities to remain living independently their own homes [2-4]. According to the World Health Organization (WHO) [5], health promotion involves structured and multicomponent actions to provide equal opportunities for the whole population to take control over their health [5]. However, previous research has visualised serious inequities with regard to the opportunities of older persons with culturally and linguistically diverse (CALD) backgrounds to achieve their fullest health potential. Poor physical and mental health among older persons with CALD backgrounds has been frequently reported in the scientific literature [6-9]. Along with documentation on cultural and linguistic barriers to healthcare access [10-12], and health promotive behaviours [13], this calls for scientific explorations on what actions to undertake in order to promote the health of an ageing and increasingly diverse population.

With increased globalisation and survival rates, confronting barriers to health for older persons with different cultural, linguistic or national backgrounds than the majority population of the country they reside in is important from a public health, as well as an ethical perspective. Access to health should be considered a human right, not to be compromised by socioeconomic or cultural factors [14, 15]. Therefore, this study is part of a larger research project with the aim of implementing a health promotion programme for ageing persons who have migrated to Sweden [16]. The aim of the present study was to systematically review randomised controlled trial (RCT) publications in order to elucidate the content and effects of multidimensional health promotion programmes that have included ageing persons with CALD backgrounds. With focus on promoting the health of all people, regardless of cultural, linguistic or national affiliation, the authors addressed the following research questions:

1. Are there any commonalities among multidimensional health promotion programmes that have included ageing persons with CALD backgrounds in the study population?

2. Are there any documented effects of multidimensional health promotion programmes on the general, mental and physical health of ageing persons with CALD backgrounds?

\section{Methods}

Cochrane [17], and PRISMA [18] guidelines were implemented to standardise the features of this systematic literature review. The PRISMA 2009 checklist is provided in Additional file 1.

\section{Selection criteria}

All included publications were screened using the following criteria as filter: 1) randomised controlled trial design, 2) participants described as ageing, older or elderly, representing a diversity of cultural, linguistic, ethnic, or national backgrounds, 3) evaluating effects of multidimensional health promotion programmes on general health, physical health and mental health. Publications were excluded if the were not written in English. Only peer-reviewed publications were included since they are commonly considered to represent the highest quality literature. No limitations with regard to year of publication were applied.

For the meta-analysis, publications were selected based on the following eligibility criteria: 1) reporting between-group differences, 2) similarity across the study arms. Publications were excluded if they reported follow-up data of another, already included, trial.

\section{Study selection}

A database search was carried out between October 2010 and February 2014 in the following databases: Amed, Cinahl, Cochrane controlled trials register, PubMed and Scopus. Assisted by a university librarian and other researchers in the field, the authors constructed chains of key words for each database in order to systematically search the variegated research field. All fields/text were searched unless indicated otherwise.

\section{Pubmed search terms}

((“culturally and linguistically diverse" OR "CALD” OR "culturally diverse" OR foreign-born" OR immigrants OR emigrants OR "immigrants and emigrants [MESH]) AND "health promotion", (limit randomized controlled trial)); ("health promotion" [MESH] AND (intervention OR "health services for the aged" $[\mathrm{MESH}]$ ), (limit randomized controlled trial)); (("emigration and immigration $[\mathrm{MESH}]$ ) OR "ethnic minority groups" OR "minority groups" OR "ethnically diverse" OR acculturation OR "cultural competence" OR "cultural congruence" OR "cultural sensitivity" OR acculturation OR multicultural OR bicultural OR intercultural OR transcultural OR "lifestyle redesign" OR "preventive home visits", (limit randomized controlled trial)); (("Ethnic groups" [MESH] AND "health promotion" [MESH], (limit randomized controlled trial)); (("lifestyle intervention" OR diversity OR "lifestyle intervention") AND health, (limit randomized controlled trial)); 


\section{Scopus search terms}

(("culturally and linguistically diverse" OR "CALD" OR "culturally diverse" [abstract, title, keyword]) AND "health promotion" [abstract, title, keyword])); ("culturally and linguistically diverse" OR "CALD" OR "culturally diverse" OR "linguistcally diverse" OR "foreign-born" OR "born abroad" [abstract, title, keyword]) AND (health AND intervention [abstract, title, keyword]); ("born abroad" AND health [abstract, title, keyword]); ((immigrants OR emigrants [abstract, title, keyword]) AND (health AND intervention AND randomized [abstract, title, keyword])); ((emigration OR immigration OR emigrants OR immigrants" OR "ethnic minority groups" OR "minority groups" OR ethnic groups") AND randomized AND "health promotion"); ("lifestyle redesign"); ((immigration OR immigrants OR emigration OR emigrants OR "ethnic minority groups" OR "ethnic groups") AND health AND randomized); (("lifestyle intervention" AND health AND randomized) NOT diabetes); (acculturation AND randomized); ((diversity OR "cultural competence" OR "cultural congruence" OR "cultural sensitivity" OR multicultural OR bicultural OR intercultural OR transcultural) AND randomized AND health)

\section{AMED search terms}

("culturally and linguistically diverse" OR "culturally diverse" OR "linguistically diverse" OR "CALD" OR "foreign-born" OR "born abroad" OR immigrants OR emigrants OR emigration OR immigration OR "ethnic minority groups" OR "minority groups" OR acculturation OR "cultural competence" OR "cultural congruence" OR "cultural sensitivity" OR multicultural OR bicultural OR intercultural OR transcultural OR "lifestyle redesign" OR "lifestyle intervention" OR "preventive home visits"); ("ethnic groups" AND health (limit clinical trial)); (diversity AND health [abstract])

\section{CINAHL search terms}

(("culturally and linguistically diverse" OR "linguistically diverse" OR "CALD” OR "lifestyle intervention" OR intercultural AND health [abstract]); ("culturally diverse" AND randomized AND health [abstract]); ("born abroad" OR emigrants OR "lifestyle redesign" OR "cultural congruence"); (("foreign-born" OR "immigrants" OR "ethnic groups" OR "minority groups" OR emigration OR immigration OR transcultural OR bicultural OR multicultural OR "cultural sensitivity" OR "cultural competence" OR diversity OR acculturation) AND (randomized OR "randomized controlled trial"))

\section{Cochrane clinical trials search terms}

("culturally and linguistically diverse" OR "culturally diverse" OR "linguistically diverse" OR “CALD” OR foreign-born" OR "born abroad” OR immigrants OR emigrants OR "immigrants and emigrants [MESH] OR "ethnic minority groups" OR "preventive home visits" OR "lifestyle redesign" OR "cultural competeonce" OR "cultural congruence" OR multicultural OR "cultural sensitivity" OR intercultural OR transcultural OR bicultural OR "preventive home visits" OR "lifestyle redesign"); (("minority groups" OR "ethnic groups") AND health); ("health promotion" [MESH] AND (diversity OR acculturation OR "health services for the aged" [MESH]); ("lifestyle intervention" AND aged $[\mathrm{MESH}])$

The first author (QL) was responsible for the literature search, screened all titles and applied the eligibility criteria in collaboration with the last author (SDI). All authors were however involved and made significant contributions to the identification of relevant publications. When there were uncertainties regarding the relevance after screening of titles, the publications' abstracts were assessed for relevancy by all authors.

\section{Data collection}

Data from all included publications were extracted using a predefined list based on Cochrane Collaboration's handbook for systematic reviews [17]. This list included: date of publication, participant data, methods, intervention details, control group details, study quality and results. For the meta-analyses, additional data on treatment effects were extracted for the following outcomes: general health, mental health, physical health, depression, and vitality. In order to be able to calculate standardised mean differences (SMD) for the selected outcomes, data on pre-test and post-test means and standard deviations (SD) were extracted, or calculated when no such data were provided in the publication.

\section{Evaluation of publication quality}

As a marker of study quality a risk of bias assessment was performed with consideration to the following aspects: randomisation (method and concealment), blinding (participants, providers, outcome assessors), dropout rates, intention-to-treat analysis, similarity of baseline characteristics, co-interventions, compliance, timing of outcome assessment [17]. Publications could score a maximum of 12 "yes" responses, and more than six "yes" responses represented a low risk of bias. All three authors were involved in the risk of bias assessment and a consensus method was employed to solve disagreements. Study authors were contacted for additional information 
when the publications did not contain enough information to assess the risk of bias.

\section{Qualitative analysis}

A narrative synthesis $[19,20]$ of extracted data on intervention details was undertaken in order to categorise the essential parts of the interventions' content into so called core components. First, all descriptions of the interventions were analysed together in order to disclose overall features. Second, an iterative process was initiated to categorise and extract the strongest features, progressively sifting out the weakest by a dialectal movement between the data and the emerging categories that represent the core components.

\section{Statistical analysis}

Random effects meta-analyses were performed with Review manager 5.3 [Revman version 5.2.8, Cochrane Collaboration, Oxford, UK] in order to estimate the average effect of health promotion on general health, mental health, physical health, depression, and vitality. SMD between intervention group participants and control group participants were calculated, and the limit for clinical relevance was set at a low level of SMD $=0.12$ in order to detect even small effect sizes that can be of importance for individual persons. Statistical heterogeneity was assessed with chisquare and $\mathrm{I}^{2}$ statistics. An $\mathrm{I}^{2}$ value of $0 \%$ indicates absence of heterogeneity, $<25 \%$ indicates low, values between 25 and $50 \%$ moderate, and $>50 \%$ high heterogeneity [21].

For evidence grading of the estimated effect on each outcome, the four level system developed by the GRADE working group [22] was used. This system ranges from level one that represents very low quality of evidence and great insecurity of results, to level four representing high quality of evidence and very small insecurity of results [22]. Considering the publications' RCT design, the grading commenced at the highest level of quality, lowered by one level for each of the following scientific considerations: risk of bias, consistency of results, directness (generalisability), and precision (sufficient data).

\section{Results}

The search process rendered a total of 9601 publications that were screened for eligibility. Following the screening procedure, 20 publications were reviewed in full-text, and finally eight publications that met the eligibility criteria were identified (Fig. 1).

Four of the included publications evaluated occupational therapy programmes with the aim to promote health [23-26], and four evaluated different means of physical activity interventions with the aim to promote health [27-30]. All programmes had community settings with lengths from six weeks up to nine months, and the intensity ranged from three sessions spread out over a

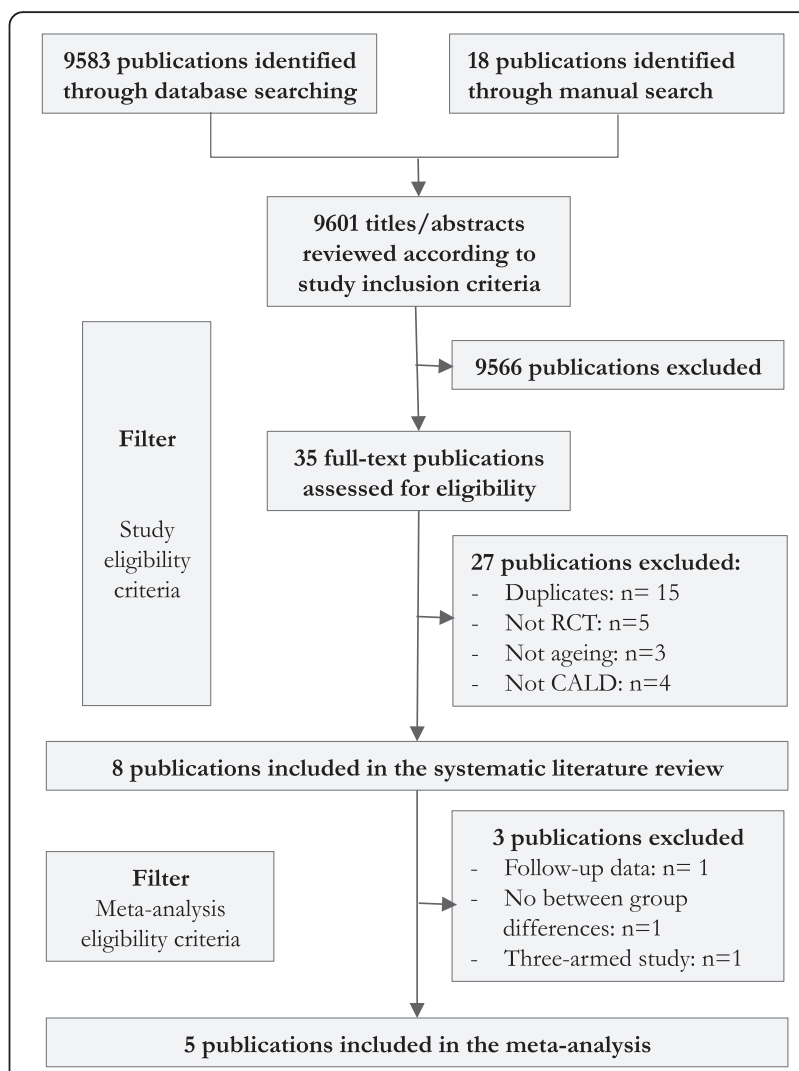

Fig. 1 Flowchart. Flowchart over the identification and inclusion of eligible publications

seven-week period, up to two hours per week over a nine-month period. A total of 1417 participants were included: 668 were randomised to health promotion and 749 were randomised to control. Women constituted $69 \%$ and men $31 \%$. The mean age was 65.3 years, ranging from 54.5 to 74.9 years. The participants' backgrounds were described in different ways: African-American (23\%), white (18\%), Asian (13\%), Hispanic (12\%), immigrants (9\%), Native American (9\%), CALD (8 \%), Mandarin-speaking Chinese (4\%), and other (4\%). Six publications were conducted in the United States $(n=1170)$, one in Australia $(n=121)$ and one in the Netherlands $(n=$ 126). More detailed information can be found in Additional file 2 .

\section{Publication quality}

The risk of bias assessment showed a range from five to nine criteria met, with seven publications having a low risk of bias [23-27, 29, 30] and the remaining [28] having a high risk of bias (Table 1).

\section{Narrative synthesis-core components of the health promotion programmes}

The narrative synthesis resulted in the following core components: activity, cultural and linguistic modifications, 
Table 1 Risk of bias assessment. Assessment of sources of risk of bias within publications

\begin{tabular}{|c|c|c|c|c|c|c|c|c|}
\hline \multirow[t]{2}{*}{ Criteria } & \multicolumn{8}{|l|}{ References } \\
\hline & $\begin{array}{l}\text { Clark et al. } \\
\text { (1997) [23] }\end{array}$ & $\begin{array}{l}\text { Reijneveld et al. } \\
\text { (2003) [27] }\end{array}$ & $\begin{array}{l}\text { Sawchuk et al. } \\
\text { (2008) [29] }\end{array}$ & $\begin{array}{l}\text { Clark et al. } \\
\text { (2001) [24] }\end{array}$ & $\begin{array}{l}\text { Borschmann et al. } \\
\text { (2000) [30] }\end{array}$ & $\begin{array}{l}\text { Clark et al. } \\
\text { (2012) [26] }\end{array}$ & $\begin{array}{l}\text { Jackson et al. } \\
\text { (2000) }[25]\end{array}$ & $\begin{array}{l}\text { Resnick et al. } \\
\text { (2008) [28] }\end{array}$ \\
\hline $\begin{array}{l}\text { 1. Adequate method of } \\
\text { randomisation? }\end{array}$ & $\mathrm{Y}$ & Y & Y & Y & $\mathrm{Y}$ & $\mathrm{Y}$ & $\mathrm{Y}$ & Y \\
\hline 2. Allocation concealment? & Y & Y & Y & Y & Y & $U$ & Y & $U$ \\
\hline 3. Patient blinding? & $\mathrm{N}$ & $\mathrm{N}$ & N & $\mathrm{N}$ & Y & $\mathrm{N}$ & $\mathrm{N}$ & N \\
\hline 4. Provider blinding? & $\mathrm{N}$ & N & N & N & N & N & N & N \\
\hline $\begin{array}{l}\text { 5. Outcome assessor } \\
\text { blinding? }\end{array}$ & Y & Y & N & Y & Y & Y & Y & $U$ \\
\hline $\begin{array}{l}\text { 6. Dropout rate described } \\
\text { and acceptable? }\end{array}$ & Y & $\mathrm{N}$ & Y & N & $\mathrm{N}$ & $\mathrm{N}$ & N & N \\
\hline $\begin{array}{l}\text { 7. All participants analysed in } \\
\text { allocated group? }\end{array}$ & Y & Y & Y & Y & $U$ & Y & $U$ & $U$ \\
\hline $\begin{array}{l}\text { 8. Free of suggestive/selective } \\
\text { outcome reporting? }\end{array}$ & Y & Y & Y & Y & $\mathrm{N}$ & Y & Y & Y \\
\hline $\begin{array}{l}\text { 9. Similarity of baseline } \\
\text { characteristics? }\end{array}$ & Y & Y & Y & Y & Y & Y & Y & Y \\
\hline $\begin{array}{l}\text { 10. Co-interventions avoided } \\
\text { or similar? }\end{array}$ & U & $U$ & U & $U$ & $U$ & $U$ & $U$ & $U$ \\
\hline 11. Compliance acceptable? & Y & Y & Y & Y & Y & Y & Y & Y \\
\hline $\begin{array}{l}\text { 12. Timing of outcome } \\
\text { assessment similar? }\end{array}$ & Y & Y & Y & Y & Y & Y & Y & Y \\
\hline Total 0-12 Y & 9 & 8 & 8 & 8 & 7 & 7 & 7 & 5 \\
\hline
\end{tabular}

$Y$ Yes, $N$ No, $U$ Unsure

More than six $Y=$ Low risk of bias

a person-centred approach, health information, and professional provision (Table 2).

\section{Activity}

All programmes included activity, albeit applied in different ways. Predominantly, the programmes had a physical approach to health promotion, with focus on: resistance exercises and stretching with instructions in lay language [28], weekly physical activity monitoring [29], low intensity exercises [27], or discussions on how to overcome individual barriers to physical activities [30]. The aim of those programmes was to increase the participants' physical activity levels to improve their health. The remaining programmes [23-26] had an occupational science approach, which means that they aimed to provide support on how health could be promoted by meaningful daily activities.

\section{Cultural and linguistic modifications}

Five programmes involved modifications in relation to the participants' cultural and linguistic affiliations. Linguistic modifications involved the use of interpreters [30], providers who could communicate with the participants in their mother tongue [23-26] and translated written

Table 2 Core components. Core components of the health promotion programmes

\begin{tabular}{|c|c|c|c|c|c|}
\hline Author, year [reference] & Activity & $\begin{array}{l}\text { Cultural and linguistic } \\
\text { modifications }\end{array}$ & $\begin{array}{l}\text { A person-centred } \\
\text { approach }\end{array}$ & $\begin{array}{l}\text { Health } \\
\text { information }\end{array}$ & $\begin{array}{l}\text { Professional } \\
\text { provision }\end{array}$ \\
\hline Borschmann et al. 2010 [30] & Physical activities & Yes & Yes & No & Yes \\
\hline $\begin{array}{l}\text { Clark et al. } 1997 \text { [23] } \\
\text { Clark et al. } 2001 \text { [24] } \\
\text { Clark et al. } 2012 \text { [26] }\end{array}$ & Meaningful activities & Yes & Yes & Yes & Yes \\
\hline Jackson et al. 2000 [25] & Meaningful activities & Yes & Yes & Yes & Yes \\
\hline Reijneveld et al. 2003 [27] & Physical activities & Yes & No & Yes & No \\
\hline Resnik et al. 2002 [28] & Physical activities & Yes & Yes & Yes & Yes \\
\hline Sawchuck et al. 2008 [29] & Physical activities & No & No & Yes & No \\
\hline
\end{tabular}


material $[25,27,28]$. Three programmes made cultural modifications to the health information [25, 27, $28]$, and two to the physical exercises according to music, dance, and instructions $[27,28]$. One programme employed the use of a peer educator from the same country as the participants when possible, and divided participants by gender according to their choice [27]. Another programme took specific consideration to cultural differences in interpersonal dynamics and norms regarding presentation of information [25]. The participants in this programme were also provided with instructions on how to deal with everyday mainstream culture issues in the society.

\section{A person-centred approach}

With focus on personal goal setting and interactive group settings four programmes [23-26, 28, 30] were considered to have a person-centred approach. Employing peer support, collective- and self-efficacy, those programmes' content took a step away from objectification, and instead regarded the participants as persons with unique experiences and needs. Through personal goal setting, the participants were strengthened in their capabilities to implement meaningful lifestyle changes. They received advice and support in their establishment of personal goals, and each person's plan was followed up throughout the programme. Interactive group settings and peer support used collective efficacy to effectively direct the participants' activities towards the achievement of their personal goals. With regard to self-efficacy, one programme [28] employed different means of strengthening the participants' confidence in their own ability and motivation for change.

\section{Health information}

Five programmes [23-29] included health information in their content to inform participants on: nutrition, benefits of physical exercise, knowledge on how to select or perform daily activities, safety in and around the participant's home, symptoms related to ageing, and how to achieve and maintain a healthy and satisfying lifestyle. The health information was provided in group settings, by occupational therapists in two of the programmes [23-26], and by a peer educator in one of the programmes [27]. In the two remaining programmes the information was provided individually through written educational materials [28], or by written education materials in combination with oral information from a research assistant [29].

\section{Professional provision}

Four of the programmes were professionally provided. Two of the programmes were provided by registered occupational therapists [23-26], one by an exercise physiologist [30], and one by a dietician [28]. The programmes delivered by occupational therapists also employed a programme specific education, and regular meetings were described to have been held between the providers and the on-site project director and manager to secure a professional provision of one of those programmes [26].

Meta-analyses of the health promotion programmes' effect Data on outcomes reported in at least three publications were analysed in order to estimate an average effect of health promotion on a variety of health related outcomes among ageing persons with CALD backgrounds.

\section{General health}

Results on general health from 566 participants in three publications $[23,25,26]$ were entered into metaanalysis, revealing a pooled effect on the rim of statistical significance ( $\mathrm{SMD}=0.17,95 \% \mathrm{CI}=-0.00$ to 0.34 ). This effect was considered clinically relevant, but considering the elevated heterogeneity $\left(\mathrm{I}^{2}=58 \%, p=0.05\right)$, and limited scientific foundation (GRADE level 2, low quality of evidence) there is more research needed in order to secure the estimations. For more detailed information and forest plot see Table 3 .

\section{Mental health}

Data from 760 participants in five publicat

ions [23-28] were pooled in meta-analysis, rendering a statistically significant and clinically relevant pooled effect on improved mental health in the intervention group $(\mathrm{SMD}=0.55,95 \% \mathrm{CI}=0.17$ to 0.92$)$. The test for heterogeneity did however uncover high heterogeneity $\left(\mathrm{I}^{2} 80 \%\right.$, $p=0.004)$, invalidating the findings. In addition, the scientific foundation is limited (GRADE level 2, low quality of

Table 3 General health. General health post-treatment, health promotion programmes versus control (results from 3 publications)

\begin{tabular}{|c|c|c|c|c|c|c|c|c|c|}
\hline \multirow{3}{*}{$\begin{array}{l}\text { Study or Subgroup } \\
\text { Clark } 1997\end{array}$} & \multicolumn{3}{|c|}{ Health promotion } & \multicolumn{3}{|c|}{ Control } & \multicolumn{2}{|c|}{ Std. Mean Difference } & \multirow{2}{*}{$\begin{array}{l}\text { Std. Mean Difference } \\
\text { IV, Fixed, } 95 \% \mathrm{Cl}\end{array}$} \\
\hline & Mean & SD & Total & Mean & SD & Total & Weight & IV, Fixed, 95\% Cl & \\
\hline & 72.8 & 19.6 & 49 & 62 & 23.2 & 110 & $25.1 \%$ & $0.48[0.14,0.83]$ & \\
\hline Clark 2012 & 46.19 & 9.85 & 187 & 45.75 & 10.48 & 173 & $68.2 \%$ & $0.04[-0.16,0.25]$ & \\
\hline Jackson 2000 & 60.6 & 22.2 & 12 & 53.4 & 27.8 & 35 & $6.7 \%$ & $0.27[-0.39,0.92]$ & \\
\hline Total $(95 \% \mathrm{Cl})$ & & & 248 & & & 318 & $100.0 \%$ & $0.17[-0.00,0.34]$ & \\
\hline \multicolumn{9}{|c|}{$\begin{array}{l}\text { Heterogeneity: } \mathrm{Chi}^{2}=4.81, \mathrm{df}=2(\mathrm{P}=0.09) ; \mathrm{I}^{2}=58 \% \\
\text { Test for overall effect: } \mathrm{Z}=1.94(\mathrm{P}=0.05)\end{array}$} & $\begin{array}{cccc}1 & 1 & 1 \\
-1 & -0.5 & 0 & 0.5 \\
\text { Favours control } & \text { Favours he }\end{array}$ \\
\hline
\end{tabular}


Table 4 Mental health. Mental health post-treatment, heatlh promotion programmes versus control (results from 5 publications)

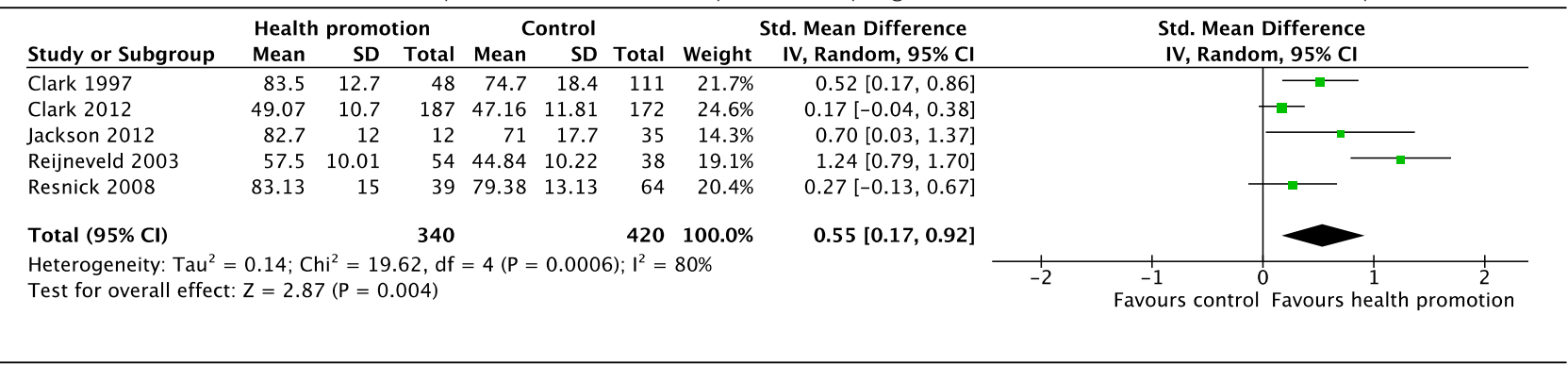

evidence). For detailed information and forest plot, see Table 4.

\section{Physical health}

Five publications [23-28] evaluated the effect of health promotion on physical health, and data from 790 participants were pooled in a meta-analysis that rendered a statistically significant and clinically relevant pooled effect in favour for health promotion $(\mathrm{SMD}=0.26,95 \% \mathrm{CI}=0.04$ to 0.49$)$. With moderate heterogeneity $\left(\mathrm{I}^{2} 50 \%, p=\right.$ 0.02 ), and low quality of evidence (GRADE level 2), the scientific foundation for the effect of health promotion on physical health is however limited. For detailed information and forest plot, see Table 5.

\section{Depression}

Data from three publications [23, 26, 28] comprising 766 participants were pooled for the effect size of depression (Table 6). The meta-analysis revealed low heterogeneity $\left(\mathrm{I}^{2}\right.$ $12 \%, p=0.007$ ), suggesting good prospects for the average pooled estimates of a significantly lower risk for depression in the intervention group $(\mathrm{SMD}=-0.22,95 \% \mathrm{CI}=-0.38$ to -0.06). Those effects were considered clinically relevant, indicating that health promotion is superior to usual care or a social intervention for depression, but the scientific foundation is limited (GRADE level 2, low quality of evidence).

\section{Vitality}

The pooled effect on vitality from three publications [23, 26,28 ] with 565 participants was just above statistical significance in favour for health promotion $(\mathrm{SMD}=0.30$, $95 \% \mathrm{CI}=0.01$ to 0.59$)$. The effect was clinically relevant, but the moderate heterogeneity $\left(\mathrm{I}^{2}=49 \%, p=0.04\right)$, and limited scientific foundation (GRADE level 2, low quality of evidence) indicate that there is more research needed to secure the effect of health promotion on vitality. For more detailed information and forest plot see Table 7 .

\section{Discussion}

This study provides a unique mapping of the content and effects of multidimensional health promotion programmes that have included ageing persons with CALD backgrounds. Visualising five core components, the findings of the review suggest a multidimensional health promotion design, and those findings are strengthened by the estimated effects on both mental and physical health revealed in the meta-analyses. Concurrent with WHO statements [31], and previous research on health promotion with older people [4, 32], the combined findings of this study encourage the inclusion of activity components and health information in health promotion programmes. What the present findings add to the scientific knowledge is a visualisation of how those two components might need to be culturally and linguistically adapted to suit the needs of an increasingly diverse ageing population. The presentation of means to bridge cultural and linguistic barriers between healthcare providers and older people with CALD backgrounds provides important information on how to make health promotion more accessible for this part of the population. However, the included publications also acknowledge hetereogeneity

Table 5 Physical health. Physical health post-treatment, health promotion programmes versus control (results from 5 publications)

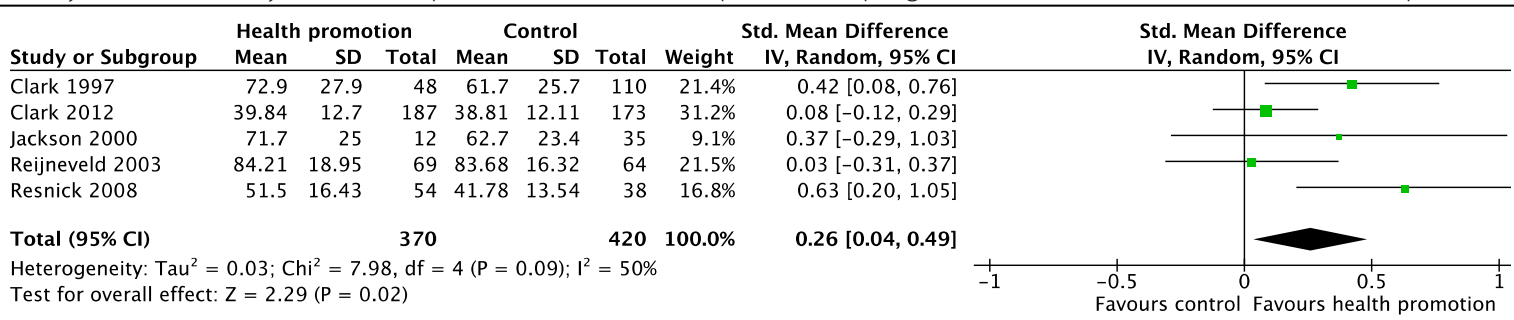


Table 6 Depression. Depression post-treatment, health promotion programmes versus control (results from 3 publications). A lower value indicates improvement for this outcome, which is why health promotion is presented to the left

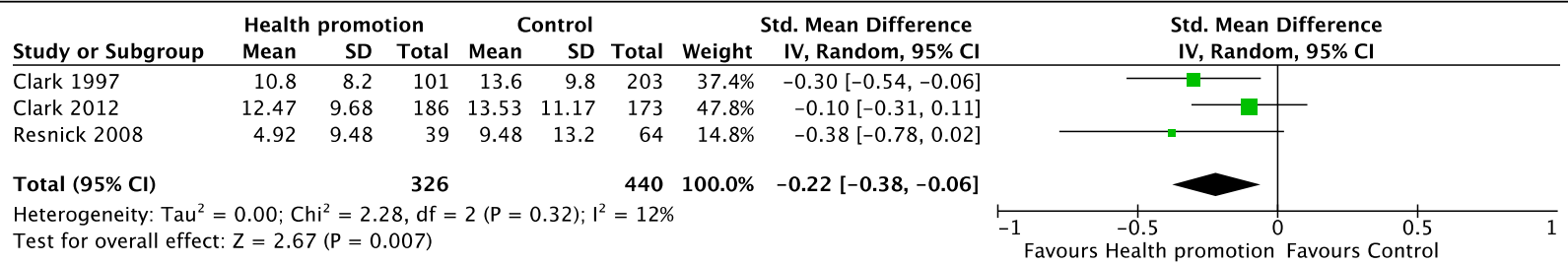

within CALD populations, and the findings support a person-centred approach to health promotion, with attention to each person's preferences and needs. As described by Turner et al. [33], person-centredness is an important part of best practice with ageing persons [33], and it is an important aspect of confronting stereotypic and stigmatising views of people with CALD backgrounds [34, 35]. Nevertheless, coming to a fore is a scientific knowledge gap with regard to how a person-centred approach could be applied to health promotion with older people with CALD backgrounds. As visualised by Hussain-Gambles et al. [36], a major problem is that researchers tend to exclude people with different cultural, linguistic, ethnic or national backgrounds in clinical trials. The present review confirms this finding, and encourages future research to put focus on the operationalisation of person-centredness into health promotion actions with an ageing and increasingly diverse population.

\section{Methodological limitations}

In the light of the present findings presented and discussed above, there are some concerns regarding the amount and quality of identified publications, as well as the heterogeneity of their participants. Therefore, the findings should be interpreted with caution, and in the light of several limitations.

First, the sparse amount of identified publications urges a questioning of the narrow inclusion criteria. The exclusion of publications on disease prevention did minimise the amount of eligible publications significantly. This was, however, considered relevant based on previous findings, which suggests that neither disease prevention nor promotion of a physically active lifestyle alone is enough to promote such a complex process as health over the ageing process $[2,3]$. The limitation of designs to only include RCT publications also narrowed down the amount of eligible publications significantly. In return, the precision of identified findings was enhanced, and the possibility to provide evidence for the programmes' efficacy improved.

Second, the majority of the reviewed trials were conducted in the United States, which makes the transferability and global application of the findings questionable. The findings show that there are commonalities across different programmes in different contexts, and that there is some scientific foundation for their efficacy. Nevertheless, consideration always needs to be given to the local contexts in which health promotion programmes ought to be implemented, and with attention to the heterogeneity of persons as well as different groups of people. Even though the meta-analyses demonstrate statistically significant effects in favour of health promotion, it is important to remember that there is no guarantee for efficacy or success in different contexts or with different populations.

Third, because data were not consistent in the publications included in the meta-analyses, it is impossible to explain to what extent health promotion programmes could contribute to improved health for older people with CALD backgrounds. In addition, the analysed interventions were carried out on small populations with heterogeneous backgrounds, and the identified publications were clinically heterogeneous. Therefore, a randomised effects model for meta-analysis was applied in order to minimise the effect of clinical heterogeneity. However,

Table 7 Vitality. Vitality post-treatment, heatlh promotion programmes versus control (results from 3 publications)

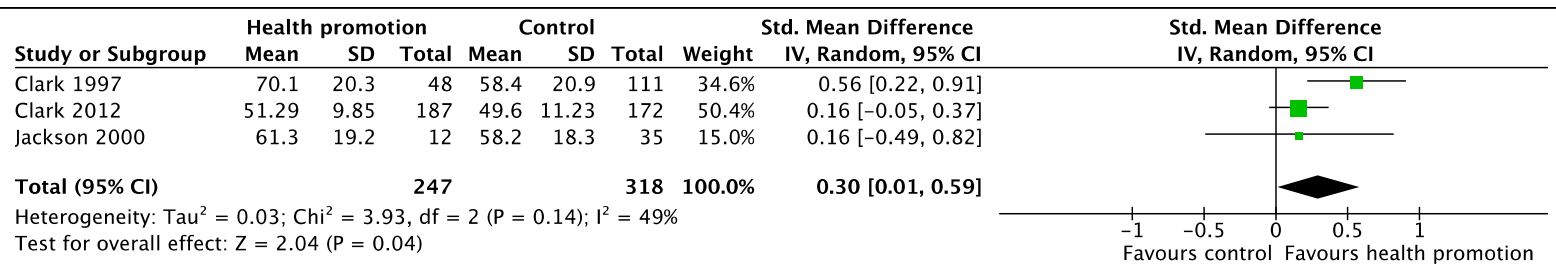


there is always a risk of drawing too generalised conclusions from a pooling of results, and the dearth of high quality RCT publications makes the evidence for the programmes' efficacy low. Where evidence is available, publications are subject to a number of methodological limitations that cloud the conclusions arising from them.

\section{Conclusions}

This study provides a unique mapping of the content and effects of health promotion programmes for ageing persons with culturally and linguistically diverse backgrounds. One of the major findings is the visualisation of how understudied this particular research field actually is. It is well known that ageing persons benefit from multidimensional health promotion programmes commenced before the onset of disease, and the visualised dearth of research with regard to ageing persons with culturally and linguistically diverse backgrounds thus poses serious threats to health equity. More research is needed in order to fully explore how to promote the health of an ageing and increasingly diverse population, and a randomised controlled trial design is suggested in order to provide ample evidence for health promotion programme efficacy.

\section{Additional files}

Additional file 1: PRISMA checklist. The PRISMA checklist used for the manuscript preparation. (PDF $358 \mathrm{~kb}$ )

Additional file 2: Data extraction form. Data extraction form. (DOC 91 kb)

\section{Abbreviations}

CALD: Culturally and linguistically diverse; CERAD: Consortium to establish a registry of alzheimer's disease; CES-D: Center for epidemiologic studies depression scale; CHAMPS: Community healthy activities program for seniors questionnaire; Cl: Confidence interval; FSQ: Functional status questionnaire; GDS: Geriatric depression scale; HAP-AAS: Human activity profile adjusted activity score; LSI-Z: Life satisfaction index-Z; MHI-5: Mental health index (from SF-36); MOS: Medical outcomes study; NRS: Numeric rating scale; OEE: Outcome expectations for exercise scale; SEE: Self-efficacy for exercise scale; SF-12: RAND short form-12; SF-36: RAND short form-36; SMD: Standardised mean difference; SOC-13: Stages of change questionnaire; WHO: World Health Organization; YPAS: Yale physical activity survey.

\section{Competing interests}

The authors declare that they have no competing interest with respect to the authorship of this manuscript.

\section{Authors' contributions}

QL participated in the design of the study, and was responsible for the study selection and data collection process, together with SDI. QL also participated in the evaluation of publication quality, performed the qualitative analyses, carried out the statisticial calculations, and drafted the manuscript. GHK participated in the study selection, data collection process and evaluation of publication quality, and confirmed the analyses. SDI was responsible for the design of the study, participated in the evaluation of publication quality, and confirmed the analyses. All authors made substantial input to the drafting of the manuscript, and have provided final approval of the submitted version.

\section{Acknowledgements}

Funding from the University of Gothenburg Centre for Person-Centred Care (GPCC 2009-1088), the Swedish Research Council for Health, Working Life and Welfare (AGECAP 2013-2300), and the Hjalmar Svensson foundation supported this work. The funders played no role in the execution or writing of the manuscript. The authors would also like to thank Ph.D. Sten Anttila for fundamental assistance with the meta-analysis, and Ph.D. Andreas Fors for lighting the spark.

\section{Author details}

IInstitute of Neuroscience and Physiology, Section for Health and Rehabilitation, The Sahlgrenska Academy, University of Gothenburg, Box 455SE-405 30 Gothenburg, Sweden. ${ }^{2}$ University of Gothenburg Centre for Person-Centred Care (GPCC), Gothenburg, Sweden. ${ }^{3}$ University of Gothenburg Centre for Ageing and Health (Agecap), Gothenburg, Sweden.

Received: 26 November 2014 Accepted: 8 December 2015

Published online: 16 December 2015

\section{References}

The 8 publications included in the review are marked by an asterisk $\left(^{*}\right)$

1. Brown P, Lang G, Resch K. Evidence-based health promotion for older people and instrumentalisation: comparing the influence of policy contexts in Austria and England. Crit Public Health. 2012:22:441-53.

2. Behm L, Wilhelmson K, Falk K, Eklund K, Zidén L, Dahlin-Ivanoff S. Positive health outcomes following health-promoting and disease-preventive interventions for independent very old persons: long-term results of the three-armed RCT Elderly Persons in the Risk Zone. Arch Gerontol Geriatr. 2014:58:376-83.

3. Gustafsson S, Eklund K, Wilhelmson K, Edberg A-K, Johansson B, Häggblom-Kronlöf $\mathrm{G}$, et al. Long-term outcome for ADL following the health-promoting RCT-elderly persons in the risk zone. Gerontologist. 2013;53:654-63.

4. Collins CC, Benedict J. Evaluation of a community-based health promotion program for the elderly: lessons from the seniors CAN. Am J Health Promot. 2006;21:45-8.

5. World Health Organization (WHO). Ottawa charter for health promotion. 1986. http://www.who.int/healthpromotion/conferences/previous/ottawa/en/. Accessed 13 Sep 2015

6. Verhagen I, Ros WJ, Steunenberg B, de Wit NJ. Ethnicity does not account for differences in the health-related quality of life of Turkish, Moroccan, and Moluccan elderly in the Netherlands. Health Qual Life Outcomes. 2014;12:138.

7. Koochek A, Montazeri A, Johansson SE, Sundquist J. Health-related quality of life and migration: a cross-sectional study on elderly Iranians in Sweden. Health Qual Life Outcomes. 2007;5:60

8. Kagawa-Singer M, Padilla GV, Ashing-Giwa K. Health-related quality of life and culture. Semin Oncol Nurs. 2010;26:59-67.

9. Liao Y, Tucker P, Giles WH. Health status among REACH 2010 communities, 2001-2002. Ethn Dis. 2004;14:S9-13.

10. Alizadeh-Khoei M, Mathews R, Hossain SZ. The role of acculturation in health status and utilization of health services among the Iranian elderly in metropolitan Sydney. J Cross Cult Gerontol. 2011;26:397-405.

11. Fiscella $K$, Franks $P$, Gold MR, Clancy CM. Inequality in quality: addressing socioeconomic, racial, and ethnic disparities in health care. JAMA. 2000:283:2579-84

12. Kim G, Worley CB, Allen RS, Vinson L, Crowther MR, Parmelee P, et al. Vulnerability of older Latino and Asian immigrants with limited English proficiency. J Am Geriatr Soc. 2011;59:1246-52.

13. Caperchione CM, Kolt GS, Mummery WK. Physical activity in culturally and linguistically diverse migrant groups to Western society. Sports Med. 2009;39:167-77

14. Braveman PA, Kumanyika S, Fielding J, Laveist T, Borrell LN, Mandersheid R, et al. Health disparities and health equity: the issue is justice. Am J Public Health. 2011:101:S149-55.

15. Commission on Social Determinants of Health (CSDH). Closing the gap in a generation: health equity through action on the social determinants of health. Final report of the commission on social determinants of health. 2008. http:/www.who.int/entity/social_determinants/thecommission/finalreport/en/ index.html. Accessed 13 Sep 2015

16. Gustafsson S, Lood Q, Wilhelmson K, Häggblom-Kronlöf G, Landahl S, Dahlin-Ivanoff S. A person-centred approach to health promotion for 
persons 70+ who have migrated to Sweden: promoting aging migrants' capabilities implementation and RCT study protocol. BMC Geriatr. 2015;15:10.

17. Higgins JPT, Green S, eds. Cochrane handbook for systematic reviews of interventions 5.1.0. 2011. http://www.cochrane-handbook.org. Accessed 13 September 2015.

18. Moher D, Liberati A, Tetzlaff J, Altman DG, PRISMA Group. Preferred reporting items for systematic reviews and meta-analyses: the PRISMA statement. PLoS Med. 2009;6:e1000097.

19. Forbes A, Griffiths P. Methodological strategies for the identification and synthesis of 'evidence' to support decision-making in relation to complex healthcare systems and practices. Nurs Inq. 2002;9:141-55.

20. Stake R. Qualitative research: studying how things work. New York: Guilford Publications Inc.; 2010.

21. Higgins JP, Thompson SG, Deeks JJ, Altman DG. Measuring inconsistency in meta-analyses. BMJ. 2003;327:557-60.

22. Atkins $D$, Best D, Peter A, Eccles M, Falck-Ytter Y, Flottorp S, et al. Grading quality of evidence and strength of recommendations. BMJ. 2004;328:1 106-10

23.* Clark F, Azen SP, Zemke R, Jackson J, Carlson M, Mandel D, et al. Occupational therapy for independent-living older adults: a randomized controlled trial. JAMA. 1997;278:1321-6.

24.* Clark F, Azen S, Carlson M, Mandel D, LaBree L, Hay J, et al. Embedding health-promoting changes into the daily lives of independent-living older adults: long-term follow-up of occupational therapy intervention. J Gerontol B Psychol Sci Soc Sci. 2001;56:P60-3.

25.* Jackson J, Kennedy BL, Mandel D, Carlson M, Cherry BJ, Fanchiang SP, et al. Derivation and pilot assessment of a health promotion program for Mandarin-speaking Chinese older adults. Int J Aging Hum Dev. 2000;50:127-49.

26.* Clark F, Jackson J, Carlson M, Chou CP, Cherry BJ, Jordan-Marsh M, et al. Effectiveness of a lifestyle intervention in promoting the well-being of independently living older people: results of the Well Elderly 2 Randomised Controlled Trial. J Epidemiol Community Health. 2012;66:782-90.

27.* Reijneveld SA, Westhoff MH, Hopman-Rock M. Promotion of health and physical activity improves the mental health of elderly immigrants: Results of a group randomised controlled trial among Turkish immigrants in the Netherlands aged 45 and over. J Epidemiol Community Health. 2003;57:405-11.

28** Resnick B, Luisi D, Vogel A. Testing the Senior Exercise Self-efficacy Project (SESEP) for use with urban dwelling minority older adults. Public Health Nurs. 2008;25:221-34.

29.* Sawchuk C, Charles S, Wen Y, Goldberg J, Forquera R, Roy-Byrne P, et al. A randomized trial to increase physical activity among native elders. Prev Med. 2008;47:89-94.

30.* Borschmann K, Moore K, Russell M, Ledgerwood K, Renehan E, Lin X, et al. Overcoming barriers to physical activity among culturally and linguistically diverse older adults: a randomised controlled trial. Australas J Ageing. 2010;29:77-80

31. World Health Organization (WHO). Active aging: a policy framework. 2002. http:/who.int/ageing/publications/active_ageing/en/index.html. Accessed 13 Sep 2015.

32. Holmberg V, Ringsberg KC. Occupational therapists as contributors to health promotion. Scand J Occup Ther. 2014;21:108-15.

33. Turner G, Clegg A, British Geriatrics Society, Age UK, Royal College of General Practitioners. Best practice guidelines for the management of frailty: a British Geriatrics Society, Age UK and Royal College of General Practitioners report. Age Ageing. 2014;43:744-7.

34. Lood Q, Dahlin-Ivanoff S, Dellenborg L, Mårtensson L. Health-promotion in the context of ageing and migration: a call for person-centred integrated practice. Int J Integr Care. 2014;14:e004.

35. Lood Q, Häggblom-Kronlöf G, Dellenborg L. Embraced by the past, hopeful for the future: meaning of health to ageing persons who have migrated from the Western Balkan region to Sweden. Ageing Soc. 2015. doi:10.1017/ S0144686X1400155X

36. Hussain-Gambles M, Atkin K, Leese B. Why ethnic minority groups are under-represented in clinical trials: a review of the literature. Health Soc Care Community. 2004;12:382-8.

\section{Submit your next manuscript to BioMed Central and we will help you at every step:}

- We accept pre-submission inquiries

- Our selector tool helps you to find the most relevant journal

- We provide round the clock customer support

- Convenient online submission

- Thorough peer review

- Inclusion in PubMed and all major indexing services

- Maximum visibility for your research

Submit your manuscript at www.biomedcentral.com/submit
() Biomed Central 